Conclusion: In our cohort, the vaccination protocol proved to be a good tool to improve the vaccination rate of rheumatological patients, despite this, the vaccination of Hepatitis $B$ and specially of influenza, continues to have a lower prevalence to general population. The vaccines were effective since none of the preventable infections occurred during follow up, despite the use of an immunosuppressant. Vaccination showed a good safety profile, without reported serious adverse effects or worsening of the underlying disease.

Disclosure of Interests: None declared

DOI: 10.1136/annrheumdis-2021-eular.1801

\section{AB0805 $\quad$ A SURVEY OF RHEUMATOLOGY PATIENTS' SATISFACTION TO SWITCHING FROM ORIGINATOR TO BIOSIMILAR AGENTS}

C. R. $\mathrm{Ng}^{1}$, S. Samec ${ }^{1}$, P. Kavanagh ${ }^{1}$, G. Mccarthy ${ }^{1}$, S. Donnelly ${ }^{1}$, A. G. Wilson ${ }^{1}$. ${ }^{1}$ The Mater Misericordiae University Hospital, Department of Rheumatology, Dublin, Ireland

Background: The recent widespread switching of patients with inflammatory rheumatic conditions from originators to biosimilars has largely been driven by costs. The views of patients on switching are also important in the successful long term switching to biosimilars. We conducted a survey of patients views on patients satisfaction with the switch to biosimilar therapy.

Objectives: To assess satisfaction and response after switching from originator (Humira or Enbrel) to biosimilar (Amgevita or Benepali respectively), and to describe efficacy, side effects and reactions to biosimilar.

Methods: All patients diagnosed with an inflammatory arthritis and switched to biosimilar were identified using the Irish national HighTech electronic prescriptions system. Participants had been administered the biosimilar for $>3$ months and were invited to take part via a telephone survey. This consisted of 4 questions (Question 1: satisfaction with the response to the new medication [Using 5 point Likert scale $=0$ : very dissatisfied; 1 : dissatisfied; 2 : neutral; 3 satisfied; 4 : very satisfied]; Question 2: overall satisfaction with biosimilar compared to originator if originator was 10 [satisfaction rating from 1 - 10 scale: 1,2 : very dissatisfied; 3,4: dissatisfied; 5: neutral; 6,7,8: satisfied; 9,10: very satisfied]]; Question 3: adverse effects with biosimilars; Question 4: opinion on usage of new device [0: very difficult; 1 : difficult; 2 : same as previous device; 3 : improvement compare to previous device).

Results: Baseline characteristics of biosimilar switch patients with disease category were:

\begin{tabular}{|c|c|}
\hline Category & (n) \\
\hline Gender & Male (39), Female (56) \\
\hline Mean age (years) & 56 \\
\hline Rheumatoid arthritis & 58 \\
\hline Psoriatic arthritis & 18 \\
\hline Ankylosing spondylitis & 16 \\
\hline Reactive arthritis & 1 \\
\hline Enteropathic arthritis & 1 \\
\hline Juvenile idiopathic arthritis & 1 \\
\hline
\end{tabular}

48 switched from Humira to Amgevita.

The majority of the patients had positive view towards the effectiveness of the biosimilar: $36 \%$ very satisfied; $28 \%$ satisfied; $13 \%$ neutral; $10 \%$ dissatisfied; $13 \%$ very dissatisfied.

$45 \%$ of the patients gave score of 9 and 10 in the survey of overall satisfaction to biosimilar if originator was given a score of 10 , followed by $32 \%$ of them gave a score from 6 to $8,4 \%$ of the patients gave a score of 5 and $7 \%$ of them gave a score of 3 and 4 . The rest of them gave a score of 1 and 2 .

12 participants switched back to originator (Humira, $n=5$; Enbrel, $n=7$ ) for the reasons of unable to use the device, anxiety, hemoptysis, nose bleeding, tongue swelling, neck pain, lethargy and generalized itchiness.

The most common complaints were (12 patients) systemic side effects (tiredness, headache, nausea, skin rashes, hair loss, muscle ache, tongue swelling, mood swing, dizzy, nose bleed, erectile dysfunction, hypertension, hemoptysis and red sclera).

$17 \%$ of the patients find the biosimilars device easier to use compare to originator and $55 \%$ of the patients find both device are similar. $24 \%$ and $4 \%$ of them find the new device is difficult to very difficult to use respectively.

Conclusion: $69 \%$ of patients from Amgevita group and $60 \%$ of patients from Benepali group were satisfied with the change. Only $28 \%$ of the patients found the new device difficult to use. Overall conclusion from the study showed less than one quarter of the participants showed dissatifaction towards biosimilar and less than $12 \%$ experienced systemic side effects and whether biosimilar could be a next cost effective biologic therapy to replace originator in future requires a longer duration of study.

Disclosure of Interests: None declared

DOI: 10.1136/annrheumdis-2021-eular.2981

\section{AB0806 \\ THE JOURNEY OF AXIAL SPONDYLOARTHRITIS IN SPAIN: FROM THE GENERAL PRACTITIONER TO THE RHEUMATOLOGIST}

E. Flores-Fernández ${ }^{1}$, C. Valera-Ribera ${ }^{1}$, I. Vázquez-Gómez ${ }^{1}$, A. V. Orenes Vera ${ }^{1}$, E. Valls-Pascual ${ }^{1}$, À. Martínez-Ferrer ${ }^{1}$, D. Ybáñez-García ${ }^{1}$, A. Sendra-García ${ }^{2}$, J. J. Alegre-Sancho'. ${ }^{1}$ Doctor Peset University Hospital, Rheumatology, València, Spain; ${ }^{2}$ Doctor Peset University Hospital, Hospital Pharmacy, València, Spain

Background: The delay in the diagnosis of axial spondyloarthropathies (AxSp), with the morbidity and economic burden that this entails, is well known ${ }^{1,2}$. According to the 2017 Atlas of axial spondyloarthritis in Spain ${ }^{3}$, the mean diagnostic delay was 8,5 years, with an average total cost per patient of $659,8 €$ including medical consultations and complementary tests until diagnosis. However, now adays there are still many patients who are incorrectly referred from the genera practitioner (GP) despite showing typical features of AxSp.

Objectives: To describe the AxSp journey until diagnosis and treatment. To analyze additional costs of either a wrong or a delayed referral of the patients with AxSp to rheumatologists.

Methods: Type of study: observational, retrospective, descriptive. We included all the patients who were referred to our Department of Rheumatology from Jan-2019 to Dec2020 and whose final diagnosis was AxSp. All the data since the first contact to the GP until the final diagnosis and initiation of treatment in Rheumatology were collected, including consultations to our emergency department $(E m D)$ and other specialists. The number of consultations, complementary tests (analytical and imaging), as well as the direct costs of all of them were also collected4. A descriptive and associative analysis of these data was carried out using the SPSS software. We used median and interquartile range (IQR) for descriptive analysis and a significant $p$ value $<0,05$. Results: From Jan-2019 to Dec-2020, 15 patients with AxSp and a median age of 43 (Interquartile range (IQR) 34-51) years were diagnosed, 10 women and 5 men. The main reason for referral was inflammatory low back pain $(66.7 \%)$. The $60 \%$ of the patients were referred from the GP, followed by the $\mathrm{EmD}(20 \%)$. Despite typical symptoms, 4 patients $(26,7 \%)$ were initially referred to Traumatology, and 3 out of them returned to the GP without the right diagnosis.

The median delay for referral from the GP to the rheumatologist was 47 (IQR $20-173$ ) days. A wrong referral of the patient was associated with a delayed diagnosis $(p 0,018)$ and higher direct costs of management $(p 0,034)$. The average cost (including medical consultation and complementary tests) of the patient referred directly to Rheumatology was 267,71 (IQR 193,7-462,3) €, while the average cost of patients referred to other specialists was 578,83 (IQR 368,32 $898,7) €$. The extra cost of a wrong referral of a patient with AxSp was $311 €$ on average per patient in our sample (Table 1)

Table 1.

\begin{tabular}{lc}
\hline Women/men & $10 / 5$ \\
Median age (years; IQR) & $43 ; 34-51$ \\
Median diagnostic delay (days; IQR) & $45 ; 20-173$ \\
Median cost of patient referred initially to Rheumatology (€; IQR) & 267,$71 ; 193,7-462,3$ \\
Median cost of patient referred initially to another specialist (€; IQR) & 578,$83 ; 368,32-898,7$ \\
Extra cost of wrong referral per patient( $($ ) & 311
\end{tabular}

Conclusion: $\mathrm{AxSp}$ is still a disease with a not negligible diagnostic delay, but it seems to be lower than previously reported. A wrong referral of the patient to other specialists, mainly Traumatology, is associated with this delay and can double the cost of managing these patients. This demonstrates the still unmet need of improving the management and referral of the patients with AxSp from the GP to the rheumatologist, ensuring an early diagnosis and treatment at the lowest cost for the system. Our study has limitations due to its small sample size, but preliminary results indicate that a larger-scale study would be necessary to correctly assess the magnitude of this problem.

\section{REFERENCES:}

[1] Fernández Carballido C. Diagnosing early spondyloarthritis in Spain: the ESPeranza program. Reumatol Clin. 2010;6(SUPPL. 1):6-10

[2] Muñoz-Fernández $S$ et al. A model for the development and implementation of a national plan for the optimal management of early spondyloarthritis: The Esperanza Program. Ann Rheum Dis. 2011;70(5):827-830

[3] Garrido Cumbrera M et al. Atlas de Espondilartritis Axial En España 2017 Vol 45.; 2017.

[4] Generalitat Valenciana. LEY 20/2017, de 28 de diciembre, de la Generalitat de tasas. [2017/12159]:96-222.

Acknowledgements: We would like to thank Novartis for its support.

Disclosure of Interests: None declared

DOI: 10.1136/annrheumdis-2021-eular.3205

\section{AB0807 TOAST-STUDY: TREATMENT AND OSTEOARTHRITIS, WHAT ARE PEOPLE SAYING ON TWITTER?}

A. Mouamnia ${ }^{1}$, A. Desvages ${ }^{2} .{ }^{1}$ Valenciennes Hospital, Rheumatology, Valenciennes, France; ${ }^{2}$ Lille University Hospital, Rheumatology, Lille, France 
Background: Osteoarthritis is a very common chronic disease. The information needs of patients vary depending on the health issue. Social media sites represent a novel source of health information and advice for patients with chronic diseases, such as osteoarthritis. Almost half of them use the internet to look for health related information [1]. No study has assessed the impact of social media on osteoarthritis and its treatment.

Objectives: The purpose of this study was to evaluate frequently discussed osteoarthritis treatments on the social media Twitter.

Methods: We retrospectively analyzed tweets, published between $1^{\text {st }}$ and $31^{\text {st }}$ January 2020, containing the keywords "osteoarthritis". Only English language tweets were included. Tweets referred to veterinary medicine were excluded. Results: 3587 tweets were analyzed. We identified 1737 tweets related to osteoarthritis treatment between $1^{\text {st }}$ and $31^{\text {st }}$ January 2020 (49.8\%) (Figure 1). Dietary interventions were the most discussed treatment $(18.3 \%, n=318 / 1737)$, including fruits, vegetables and plants $(n=101)$, dietary supplements and vitamins $(n=80)$, and spices $(n=19)$. Physical medicine and rehabilitation $(17.6 \%, n=305 / 1737)$ including sport $(n=151)$, physiotherapy $(n=70)$ and rehabilitation $(n=57)$, were commonly discussed. Local therapies for osteoarthritis were also discussed by Twitter users (15.8\%, $n=274 / 1737)$. These included topical treatments such as anti-inflammatory gels and creams $(n=23)$, and more invasive local treatments including intra-articular joint injections with corticosteroid $(n=56)$, hyaluronans $(n=29)$, stem cells $(n=97)$, and Platelet-Rich Plasma $(n=52)$. The frequently used systemic drugs were analgesia and non-steroidal anti-inflammatory drugs $(n=113)$. Surgery and interventional radiology (genicular artery embolization) were also discussed (11.5\%, $n=199 / 1737) .5 .6 \%$ tweets $(n=97 / 1737)$ were related to alternative therapies. Predominant themes were related to marijuana $(n=23)$, acupuncture treatment $(n=17)$, homeopathy $(n=10)$. Last but not least, 356 tweets $(20.5 \%)$ referred to other websites including health programs.

Conclusion: Our results demonstrate that osteoarthritis treatment is frequently discussed in published tweets. Thereby, social media could have an impact on behaviors and adherence on medication, and it seems interesting that learned societies, involved in osteoarthritis treatment, communicate more using social media.

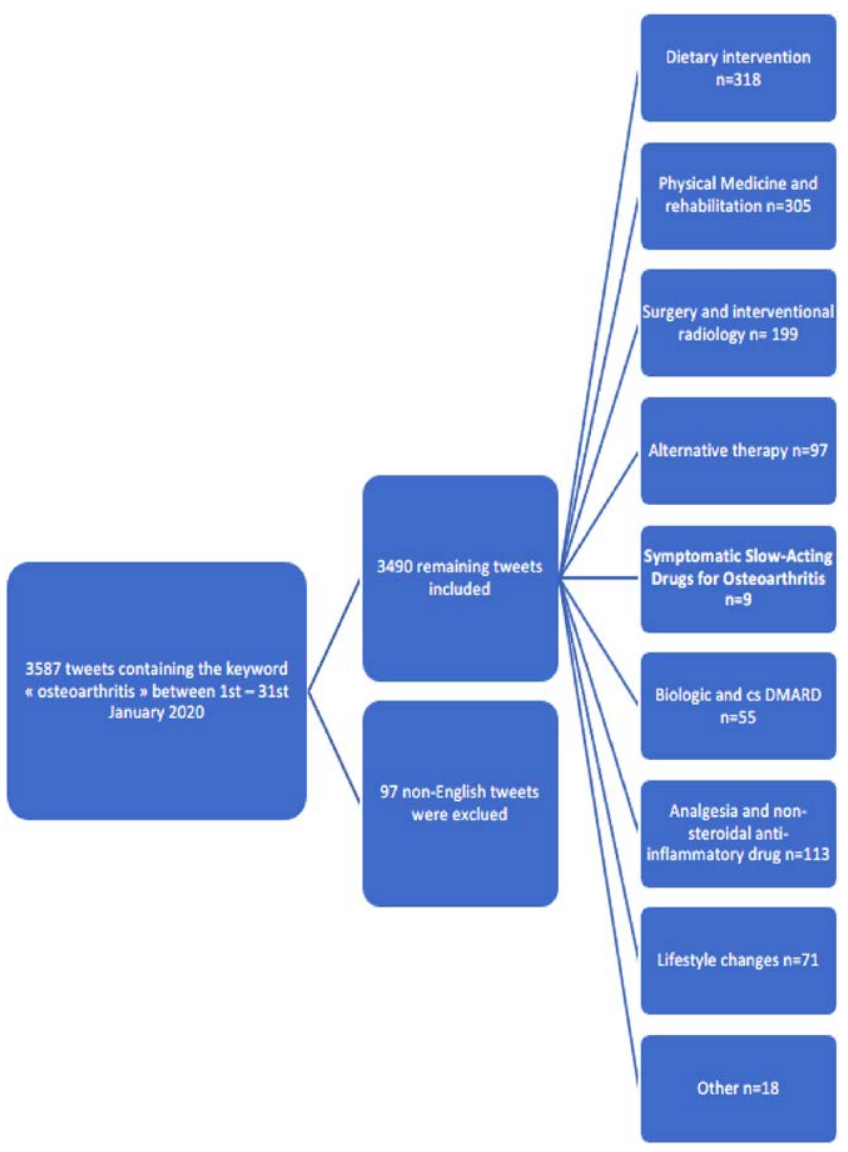

Figure 1. Osteoarthritis treatment discussed in published tweets

\section{REFERENCES:}

[1] Wagner TH, Baker LC, Bundorf MK, Singer S. Use of the Internet for health information by the chronically ill. Prev Chronic Dis 2004;1(4):A13.

Disclosure of Interests: None declared

DOI: 10.1136/annrheumdis-2021-eular.3299

\section{\begin{tabular}{|l|l}
\hline AB0808 & A SYSTEMATIC REVIEW ON SYNDEMICS IN
\end{tabular} RHEUMATIC AND MUSCULOESKELETAL DISEASES (RMDS)}

M. F. Ramirez-Flores ${ }^{1}$, C. Cadena-Trejo ${ }^{2}$, D. Alpizar-Rodriguez ${ }^{3}$,

V. Fernandez-Garcia ${ }^{4}$, A. Gastelum-Strozzi ${ }^{5}$, A. Hernandez-Garduno $^{6}$,

I. Peláez-Ballestas ${ }^{7}$ on behalf of Mexican Syndemic Study Group.

${ }^{1}$ Universidad Nacional Autónoma de México, Plan of Combined Studies in

Medicine (PECEM-MD/PhD), Mexico City, Mexico; ${ }^{2}$ Universidad Nacional

Autónoma de México, Escuela Nacional de Enfermetía y Obstetricia,

Mexico City, Mexico; ${ }^{3}$ Colegio Mexicano de Reumatología, Research

Unit, Mexico City, Mexico; ${ }^{4}$ Escuela Nacional de Enfermería y Obstetricia,

Universidad Nacional Autónoma De México, Research Unit, Mexico City,

Mexico; ${ }^{5}$ Instituto de Ciencias Aplicadas y Tecnolog ía, Universidad Nacional

Autónoma de México, Scientific and Industrial Instrumentation, Mexico City,

Mexico; ${ }^{6}$ Private Practice, Private Researcher, Mexico City, Mexico; ${ }^{7}$ Hospital

General de México “Dr. Eduardo Liceaga”, Rheumatology, Mexico City,

Mexico

Background: Syndemics comprises the interaction of two or more biological diseases in different socio-cultural situations and in the context of varying healthcare standards that exacerbates their deleterious effects on the health of individuals, communities, and societies (1). There is increasing interest in using syndemics in the study of RMDs.

Objectives: To identify quantitative and qualitative RMDs studies focused on syndemics through a systematic review and synthesize the available evidence.

Methods: Inclusion criteria were using a syndemic framework in methods in RMDs studies. A systematic search of studies published from 2003 up to January 2021 was conducted in two electronic databases (SCOPUS and Web of Science). The systematic search was implemented (Figure 1): 1) the word syndemic(s) was searched using the advanced query string search, in the title, abstract, keywords, and text, to identify the relevant studies; 2) the publications were screened by three peer review groups; 3 ) the group evaluated each title and abstract and reviewed full-text articles to identify those relevant for review; 4) synthesis focused on identifying the variables of each study and integrating findings.

Results: The initial search yielded 658 unique articles, from which ten studies were identified as syndemics in RMDs. After full-text review, six studies were excluded due to not inclusion of a syndemic framework. Four studies were included (Figure 1). The first quantitative study was on eight Latin-American (LatAm) indigenous communities $(n=6,155)$. RMD's were highly prevalent in the indigenous people (34.5\%), RA prevalence was $2.4 \%$ in the Qom group of Argentina, and low back pain (LBP) being the most prevalent RMD (13.3\%). RMDs were strongly associated with other comorbidities, unhealthy habits, low education levels, rural areas, socioeconomic conditions, and health inequality. The network analysis was the analytical approach using the Syndemic and Syndemogenesis Elements Modeler (SSEM). The second quantitative study focused on six urban/rural LatAm countries $(n=55,724)$. LBP was significantly associated with comorbidities, unhealthy habits, low educational level, rural areas, and indigenous status. SSEM and cluster analysis showed a significant association between LBP prevalence and social variables. One qualitative study in fisher rural families $(n=7)$ with three fisher rural generations from the Netherlands. A case study using life course theory. The first two generation reported syndemic vulnerability (psychological distress, cardiometabolic conditions, or musculoskeletal pain), and the third generation report less syndemic vulnerability, including musculoskeletal pain. A mixed study was identified focused on osteoarthritis with multimorbidities (cardiovascular, hypertension, diabetes mellitus) in an African American population $(n=110)$ in the USA. The methodology was a descriptive secondary analysis and qualitative interviews. The study found that patients with hypertension $(80.9 \%)$ reported greater chronic pain than those without. The lack of education added to the incorrect use of nonsteroidal anti-inflammatory drugs might contribute to the worst outcomes in this population, a significant burden of cardiovascular disease.

Conclusion: There is growing interest and urge for integrating syndemics in the study of RMDs. This review has demonstrated that there are only a few publications to date. The statistical power and the analytical approach (SSEM-Syndemic and Syndemogenesis Elements Modeler) in the two quantitative studies are relevant. The qualitative study demonstrated less syndemic vulnerability with better health conditions linked to better socioeconomic advantages. The mixed qualitative study did not show a syndemic framework. It is necessary to carry out studies in RMDs from the syndemic perspective to document the complexity of the clinical and social determinants related to these diseases.

REFERENCES:

[1] Singer M, Bulled N, Ostrach B, et al. Syndemics and the biosocial conception of health. The Lancet 2017;389:941-50. 